\title{
Orthobiologic Supplementation Improves Clinical Outcomes Following Lumbar Decompression Surgery
}

\author{
Solomon Kamson ${ }^{\mathrm{a}, \mathrm{c}}$, DuPreez Smith ${ }^{\mathrm{b}}$
}

\begin{abstract}
Background: Endoscopic-assisted lumbar decompression is a minimally invasive spine surgery which has been touted to reduce collateral tissue damage, incisional pain, recovery time and complications. Residual back or leg pain and recurrent herniation are commonly reported post-operative outcomes. It has been suggested that injecting orthobiologics like cryopreserved amniotic-derived products (ADPs) and bone marrow aspiration (BMA) into the surgery site would have additional benefit on patient outcomes. This is a Western Institutional Review Board (WIRB)-approved level 1, randomized controlled trial of prospectively collected patient demographic and outcomes data for endoscopic-assisted lumbar decompression surgery. The primary goal of this study was to compare patient outcomes of orthobiologic supplementation during endoscopic-assisted lumbar decompression surgery.
\end{abstract}

Methods: Following WIRB approval, 269 patients underwent lumbar endoscopic-assisted decompressive surgery between January 2011 and October 2017. Patients were randomized to receive ADP, BMA, both, or no supplementation (control group). Outcomes were measured by post-operative questionnaires (visual analog scale (VAS), Oswestry disability index (ODI), 36-item short-form health survey (SF-36)) over 12 months.

Results: Mean VAS-leg for either BMA or ADP group displayed statistically significant improvements at 2 weeks (3.55 vs. 4.77, P $=0.002), 6$ months $(2.34$ vs. $3.37, \mathrm{P}=0.026)$, and 9 months $(2.18$ vs. $3.57, \mathrm{P}=0.01$ ) compared to no supplementation group (control group). Similarly, improvements in mean VAS-back were significant at 2 weeks (3.98 vs. 5.01, $\mathrm{P}=0.011), 2$ months (3.22 vs. 3.93 , $\mathrm{P}=0.04), 9$ months $(2.38$ vs. $4.11, \mathrm{P}=0.004)$, and 12 months $(2.23$ vs. $3.58, \mathrm{P}=0.011$ ) compared to no supplementation group (control group). There were statistically significant differences in the ODI ( 2 weeks, 42.19 vs. $31.11, \mathrm{P}=0.014$ ) and SF-36 (4 months, 85 vs. $63, \mathrm{P}=0.043$ for ADP only), but these differences did not subsist over time. Two patients (one control, one ADP) re-herniated at the

Manuscript submitted November 4, 2019, accepted November 20, 2019

aspine Institute Northwest, Bothell, WA, USA

${ }^{b}$ Royal College of Surgeons, Dublin, Ireland

${ }^{\mathrm{c} C}$ Corresponding Author: Solomon Kamson, Spine Institute Northwest, 1629

220th St SE, Bothell, WA 98021, USA.

Email: research@spineinstitutenw.com

doi: https://doi.org/10.14740/jocmr3972 same level.

Conclusions: Orthobiologic BMA and ADP resulted in improved pain control during early post-operative periods.

Keywords: Endoscopic discectomy; Lumbar discectomy; Spinal stenosis; Bone marrow aspiration; Cryopreserved amniotic products; Epidural scarring; Post-operative pain; Orthobiologic supplementation

\section{Introduction}

Endoscopic-assisted lumbar decompression is a minimally invasive spine surgery which has been touted to reduce collateral tissue damage, incisional pain, recovery time and complications [1-3]. Endoscopic-assisted lumbar decompression to treat disc herniation-induced radicular pain is generally considered the standard of care when reasonable course of non-surgical care has failed to alleviate symptoms. Most patients have good relief, but some have persisting pain. It is thought that the pain is associated with the neuro-inflammatory pathways.

Neuro-inflammation is associated with neuropathic pain syndrome which has characteristic features. These include infiltration of immune cells into the peripheral nervous system such as the dorsal root ganglia (DRG), activation of microglia and astrocytes, and production of pro-inflammatory cytokines such as tumor necrosis factor (TNF), interleukin (IL)-1 $\beta$, and IL-6. Abundant research has suggested that this inflammatory response can be mediated by transforming growth factor- $\beta 1$ (TGF- $\beta 1$ ). There is also evidence to suggest that the modulation of inflammation is possible by inhibiting monocyte infiltration, activation of the glia, and cytokine production in the DRG and spinal cord. In essence, it is through paracrine signaling, a type of cell-to-cell communication, that inflammation may be controlled by biological intervention.

Two common biologic approaches to modulating inflammation have emerged over the last several years. One is using amniotic-derived product (ADP) and the other is bone marrow aspiration (BMA)-containing messenger signaling cells (MSCs). The mechanisms of action are not altogether different. ADP is thought to exhibit anti-inflammatory properties reducing pro-inflammatory cytokines, increasing anti-inflammatory cytokines, and inducing apoptosis of pro-inflammatory cells [4-6]. Bone marrow-derived stem cells have been examined in a number of applications for their ability to modulate neuro-in- 


\section{Patient Supplementation Distribution}

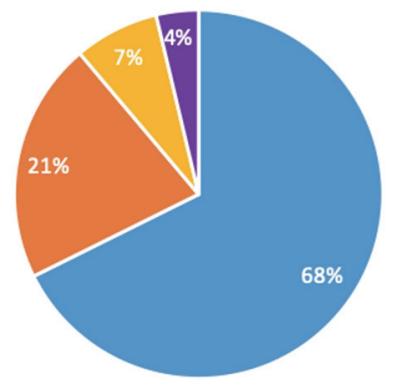

\section{- Control \\ - Amnio \\ $\because \mathrm{BMA}$ \\ - Both}

Figure 1. Patient supplementation distribution.

flammation. They have been delivered via intra-ganglionic, intra-spinal, intra-brain, intramuscular and intrathecal injection as well as through intravenous infiltration. It has suggested that this inflammatory response can be mediated by TGF- $\beta 1$. There is also evidence to suggest that the modulation of inflammation is possible by inhibiting monocyte infiltration, activation of the glia, and cytokine production in the DRG and spinal cord.

In the present study we sought to determine the clinical efficacy of BMA-containing MSCs and growth factors and ADP in reducing post-operative pain in a population of minimally invasive endoscopic-assisted surgery patients.

\section{Materials and Methods}

This research is Western Institutional Review Board (WIRB)approved level 1 study. This study was conducted in accordance with the ethical standards of the responsible institution on human subjects, as well as with the Helsinki Declaration. Informed consent was taken from patients prior to their participation in this randomized control trial.

\section{Gender Ratio for Supplementation Type}

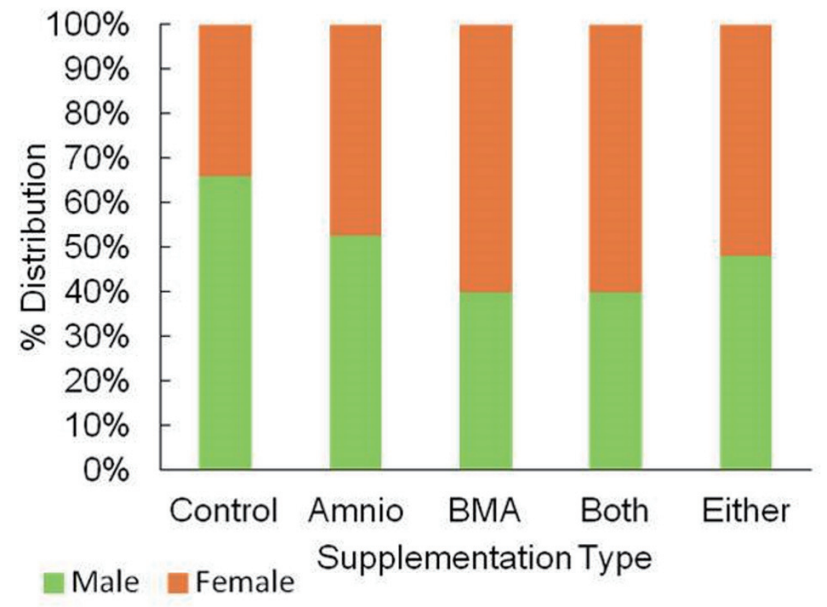

Figure 2. Gender ratio for supplementation type.

\section{Age Distribution}

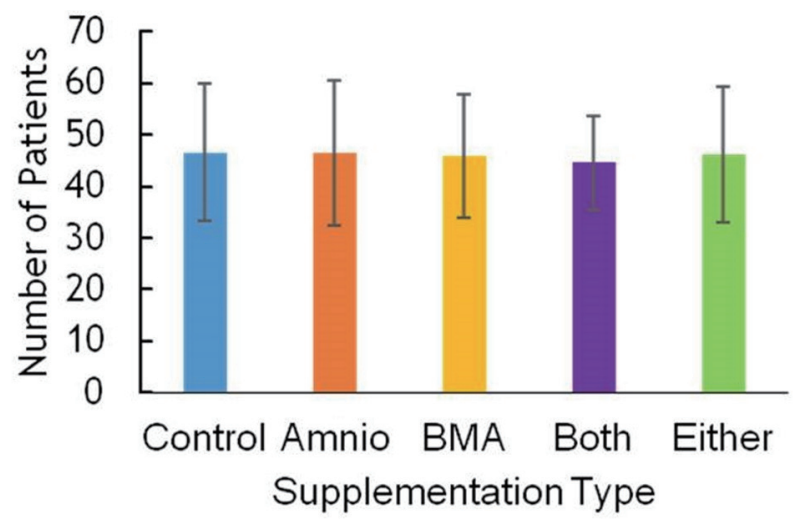

Figure 3. Age distribution.

\section{Patient characteristics}

Following WIRB approval, 269 patients underwent lumbar endoscopic-assisted decompressive surgery between January 2011 and October 2017. The patients included in our study were healthy adults between the ages of 16 and 90. These patients had to be conversant in English, and able and willing to comply with all study requirements. Each patient was able to provide written consent to participate in this study and was willing to undergo surgery with the ADP and BMA supplemented at the end of the procedure. The exclusion criteria for our study were as follows: no pediatric patients were included, nor patients who were pregnant, lactating, or had the intention to become pregnant. Patients who were immune-compromised, or who were due to be undergoing immunosuppressive therapy, were not included in this study. Any patients with a history of allergies, or any other contraindications, that could be triggered by the therapy in this study were not included. Patients who had participated in other clinical trials within 12 weeks of this study were not allowed to participate.

Patients were randomized to receive amniotics, BMA, both, or no supplementation (Fig. 1). Only the patient could be blinded to treatment assignment. Of the total 269 patients, the majority was male, with 162 men versus 107 women (Fig. 2 ). The age range for patients in this study was between 16 and 90 years of age, with the majority being 30 to 60 years old, and having a mean of 46.4 years (Fig. 3). The average body mass index (BMI) of patients was $29.7 \mathrm{~kg} / \mathrm{m}^{2}$ and ranged from 17.6 to $37.2 \mathrm{~kg} / \mathrm{m}^{2}$ (Fig. 4). Outcomes were measured by postoperative questionnaires (visual analog scale (VAS), Oswestry disability index (ODI), and 36-item short-form health survey (SF-36)) over 12 months (Figs. 5-8). The patients included in the study presented with unmanageable back and leg pain. Data regarding the race of patients was not recorded.

In $97 \%$ of patients, lumbar degenerative disc disease was a presenting diagnosis. Facet arthropathy (82\%), foraminal stenosis $(72 \%)$, and central canal stenosis (57\%) were other common clinical presentations. All of the patients failed conservative treatment for at least 6 months and through magnetic resonance imaging (MRI) and computed tomography (CT) im- 


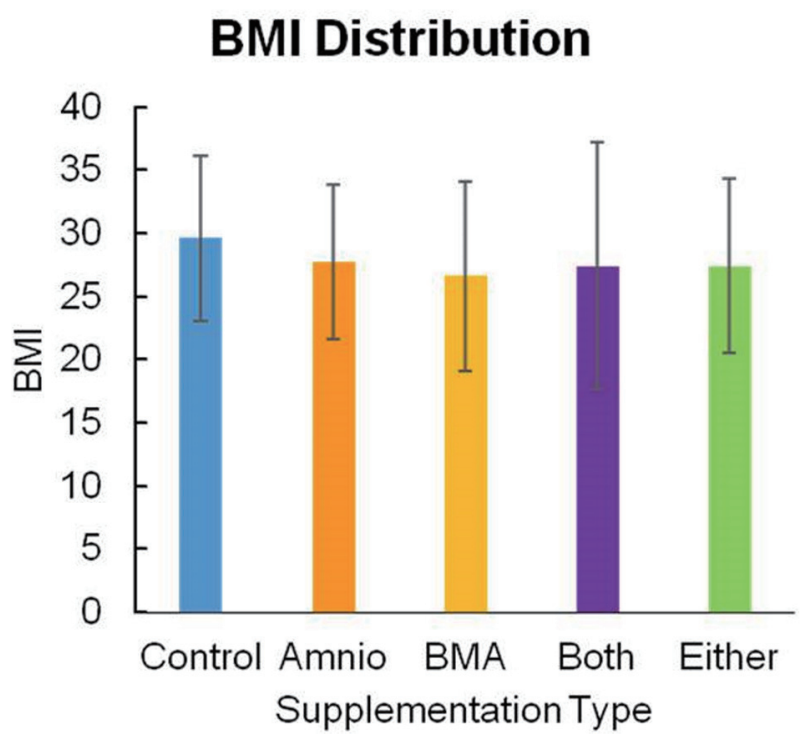

Figure 4. BMI distribution. BMI: body mass index.

aging their nerve compressions were confirmed.

\section{Surgical setting}

This evidence-based level 1 study took place in a Joint Commission certified free-standing, out-patient same-day surgery center.

\section{Anesthetic technique}

For each surgery, monitored anesthesia care (MAC) was used. No patients in this study underwent general endotracheal anes- thesia. All patients were placed on a Kambin frame in a prone position while being prudently monitored by an anesthesiologist. During surgery, supplemental oxygen was supplied through nasal prongs or a face mask. Utilizing regional anesthetic technique, intra-spinal narcotic medication was given in conjunction with light intravenous supplementation. Extremity movement on verbal command was documented and a wake-up test, for neurophysiological monitoring, was performed in every case. This technique is proven very effective due to the instant and direct feedback from patients. Patients are instructed to verbalize immediately in response to a painful event which provides valuable information on the surgical site. Through all clinical trials, no patients were put under general anesthesia or converted to this technique. In addition, no patients experienced complete motor loss due to the local anesthetic.

\section{Surgical technique}

In order to access the affected areas of the spine, full-endoscopic transforaminal and inter-laminal techniques were applied. This method is a minimally invasive approach and has been certified as standard protocol [7-11]. Using a 4-mm endoscopic working tube retractor with trephines, Kerrison, Ronguer, and power burs, we were able to resect bone with direct visual feedback from our monitors and under complete control. In cases where the pathological entities are inoperable through the transforaminal technique, we used the inter-laminal approach [8]. This awake and awareness anesthetic technique is unique compared to most lumbar spine surgeries which utilize the inter-laminal midline dissection with patients under general endotracheal anesthesia. For increased accuracy in targeting specific pain sites in patients, bi-planar radiological imaging was used. Following an incision into the ligamentum flavum with exposure of the underlying neural structures, transforami-

\section{Leg Visual Analog Pain Scale}

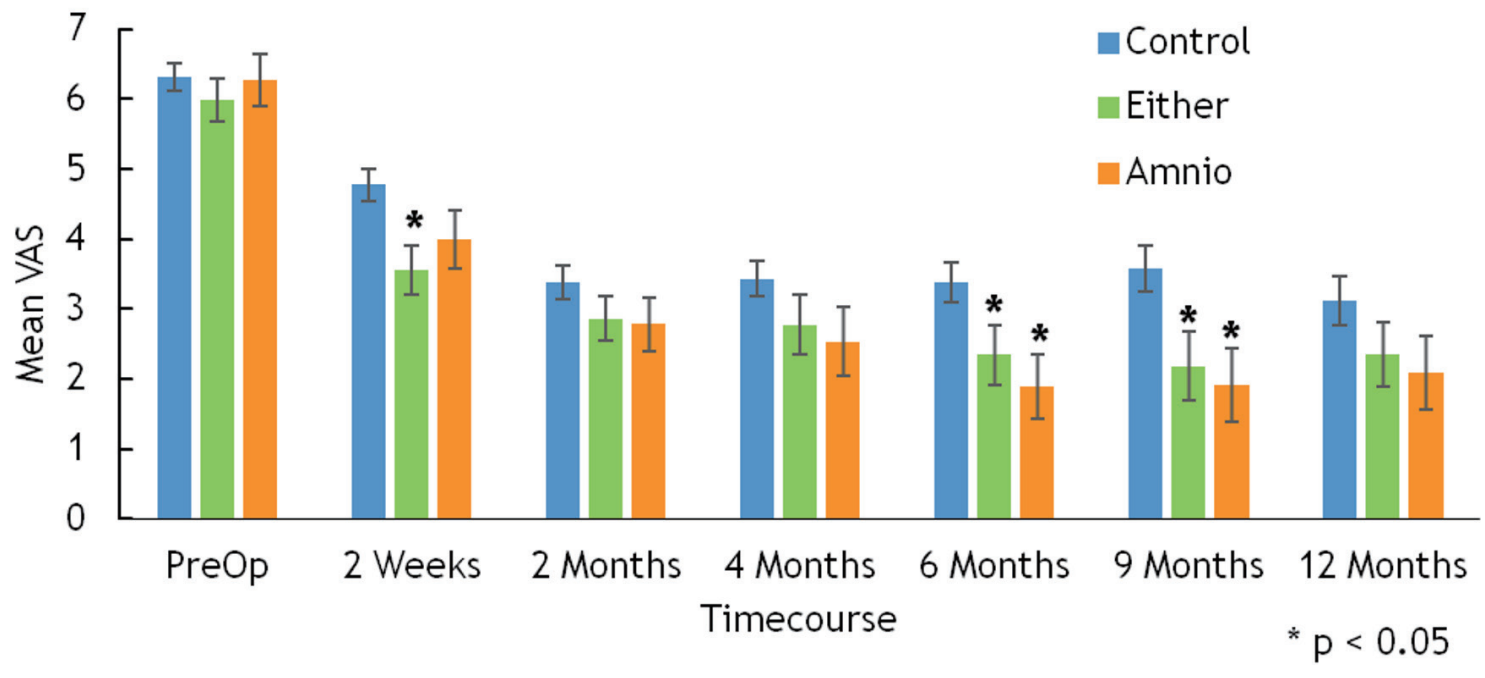

Figure 5. Leg visual analog pain scale. 


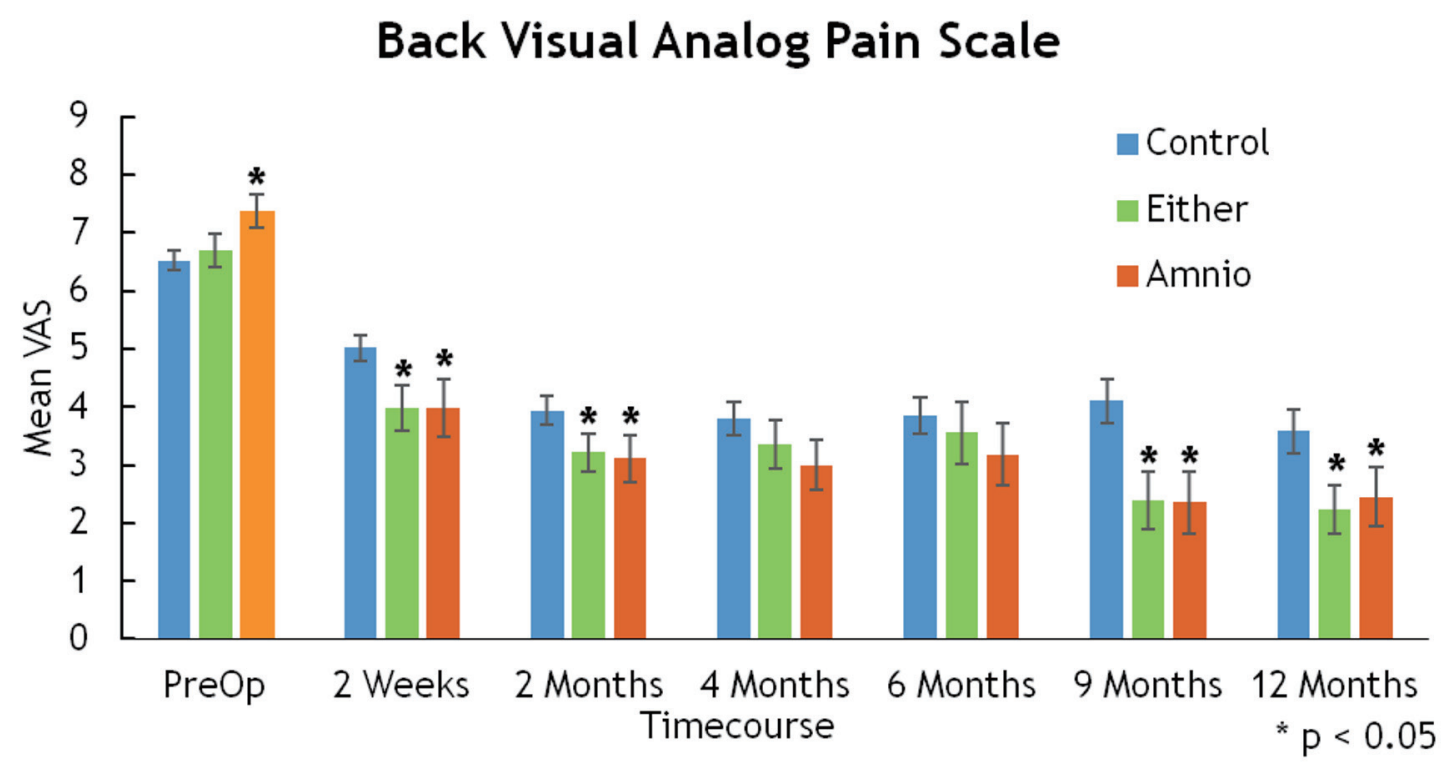

Figure 6. Back visual analog pain scale.

nal and inter-laminal approaches were implemented under direct visual control with constant irrigation. The beveled end of the endoscope can be rotated and used as a safety mechanism to avoid damaging nearby nerves. With this instrument, we also have completely controlled optics, with cranial and caudal mobility. Once inserted in the lateral recess, full rigid lens endoscopic instrument provides a great degree of freedom to visualize the foraminal, medial, lateral, and central epidural space (Fig. 9).

Surgical dissection involves performing medial facetectomy with power burs and annulotomy to remove herniated disc fragments inter-positioned in annular tears. Traversing and exiting nerve roots are protected by the working tube hood (Fig. 10). Once annular defects are cleared of fragments, using pituitary Rongeur, radiofrequency (RF) heat probe is used to shrink the annular defect prior to injection of supplementation products (Fig. 11). Following injection of orthobiologic supplementation products, the retraction tube was removed and skin was closed with 3-0 non-absorbable suture.

Patients randomized to the control arm of the study underwent the same procedure without the added step of orthobiologic supplementation products.

\section{ADPs \& BMA}

ADPs arise from the same cellular origin as the developing fetus and are a placental-derived tissue [12]. In the past, ADPs

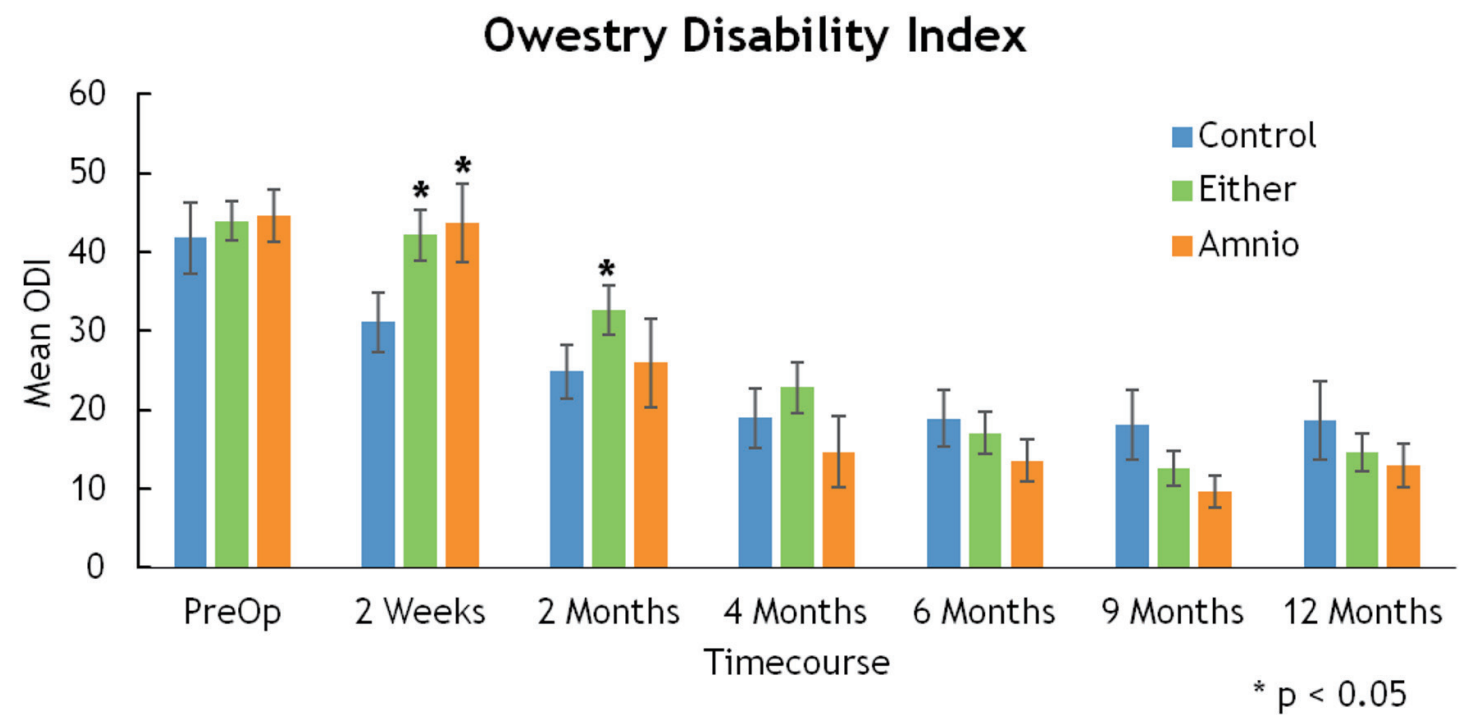

Figure 7. Owestry disability index. 


\section{SF-36 Physical}

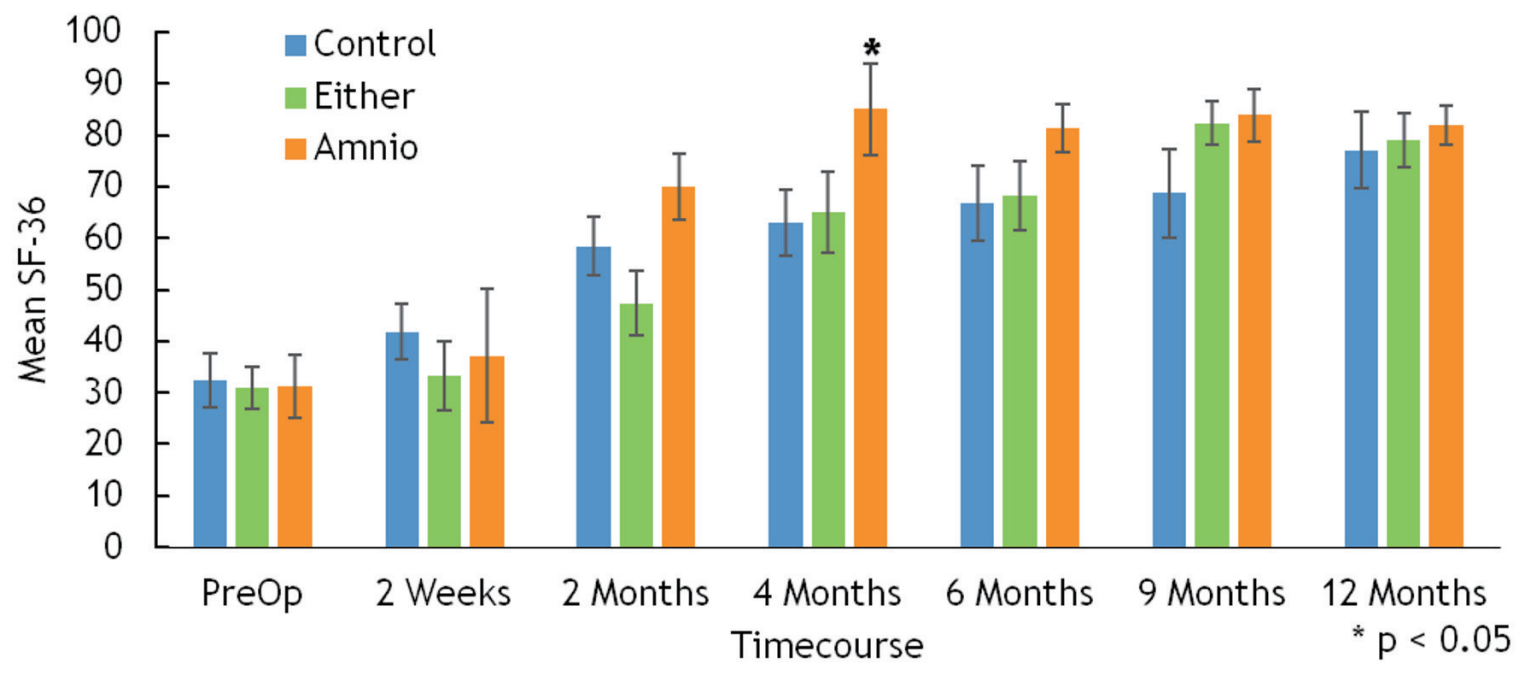

Figure 8. SF-36 physical. SF-36: 36-item short-form health survey.

have been used in the field of ophthalmology [13], as wound dressing for burns, treatment for problematic skin ulcers, and promoting of wound healing [14-16]. ADPs have demonstrated strong anti-inflammatory properties [4], supporting the theory that the main function of the amniotic membrane is to protect the fetus against the maternal immune system [12]. Moreover, ADPs have the capacity to cause apoptosis or cell death of proinflammatory cells while also reducing pro-inflammatory cytokines and increasing anti-inflammatory cytokines $[5,6]$. Another beneficial characteristic of ADP is the anti-scarring properties it has displayed $[17,18]$.

In addition to ADP, we also supplemented patients with bone marrow harvested from the iliac crest containing MSCs. These BMA contents are useful due to their ability to differentiate into multiple cell types [19]. BMA-containing MSCs have been proven to ease early and late phase neuropathic pain symptoms, such as allodynia and hyperalgesia, for several weeks post-surgery [20]. Furthermore, MSCs decreased

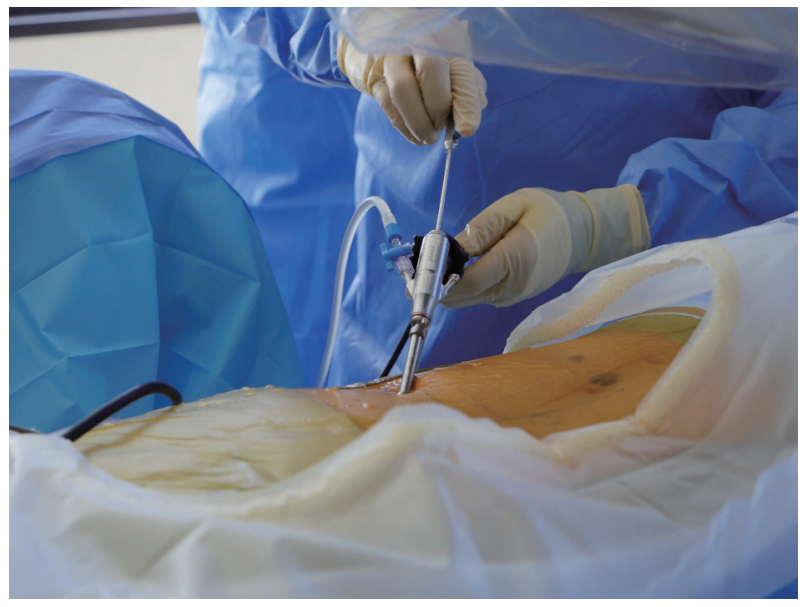

Figure 9. Endoscopic discectomy. chronic constriction injury (CCI)-induced spontaneous pain and axonal injury of the dorsal root ganglion while also inhibiting CCI-evoked neuroinflammation [20].

\section{Measuring instrument}

A VAS is used to measure a characteristic or psychometric response to a certain stimulus which is believed to span across a continuum. During this study, before patients underwent surgery, they were asked to rate their back and leg pain using a VAS. Factors such as estimated blood loss, perioperative complications, and operating and post-operative recovery time were recorded by physicians or nurses. Patients were assessed either in the clinic or through a phone call at 1 day, 2 weeks, 2 months, 4 months, 6 months, 9 months, and 1 year post-operation. At each of these checkpoints, patients were asked to rate their leg and back pain using a VAS which was included in a post-operative questionnaire. Patients were also asked to

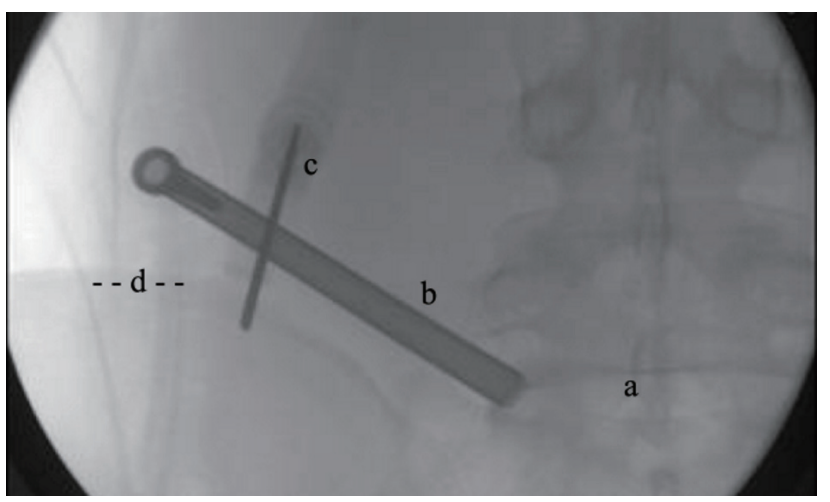

Figure 10. BMA harvesting-intraoperative X-ray. a: intervertebral disc; b: access tube; c: Jamshidi aspiration needle; d: posterior superior lliac Spine. BMA: bone marrow aspiration. 


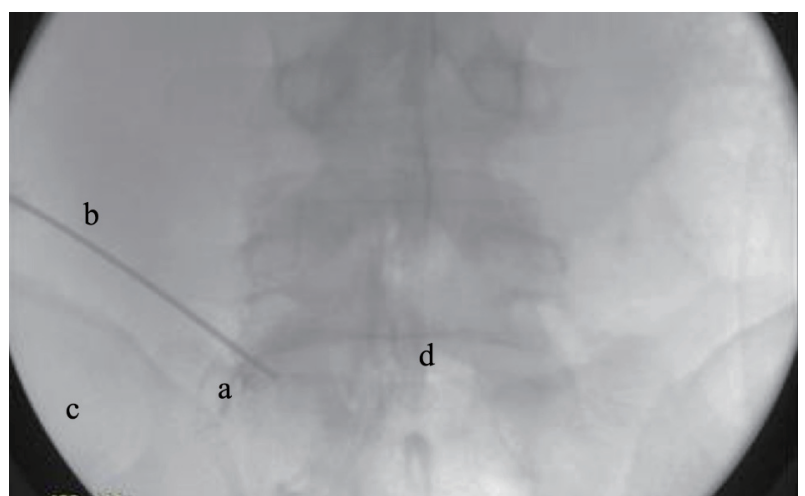

Figure 11. Injection of supplementation. a: annulotomy defect filling; b: needle; c: PSIS; d: interverterbral disc. PSIS: posterior superior iliac spine.

complete a satisfaction survey regarding the surgery and their quality of life.

\section{Literature review}

A computerized search for relevant and past research regarding this topic was conducted. Various peer-reviewed original studies, literature reviews, and case reports were accessed and considered through PubMed and the Royal College of Surgeons in Ireland (RCSI) library database (RCSI, Dublin, Ireland). Keywords such as "neuropathic pain", "mesenchymal stem cells", "herniation", "microdiscectomy", "bone marrow stromal cells", "cellular therapy," and "stem cell transplant" were used in the search to find related articles. The language was restricted to English.

\section{Results}

\section{Estimated blood loss}

About $95 \%$ of patients had less than $5 \mathrm{~mL}$ of blood loss. No patients lost more than $35 \mathrm{~mL}$ of blood during surgery.
Factors affecting operating room (OR) time and postanesthesia care unit (PACU) time

\section{OR time}

A non-linear regression of operating time on age, gender, BMI, and indicators of degenerative disc and central canal stenosis results in a model explaining $33 \%$ of the variation of OR time $(\mathrm{R} 2=0.33)$.

Age is a significant predictor of OR time; older patients generally had longer surgeries (Fig. 3). BMI does not have statistically significant effect on OR time (Fig. 4). As indicated in Table 1, the coefficients of degenerative disc disease and central canal stenosis suggest that presence of degenerative disc increases OR time by $38 \mathrm{~min}$, whereas patients with central canal stenosis have a 16-min increase in their OR time.

\section{PACU time}

A non-linear regression of operating time on age, gender, and BMI results in a model that explains $9 \%$ of the variation of OR time $(\mathrm{R} 2=0.09)$. Table 2 shows the results of statistical analysis.

Based on this model, gender, age, and BMI do not appear to be statistically significant $(\mathrm{P}>0.05)$ to $\mathrm{PACU}$ time.

\section{VAS score assessment}

Mean VAS-leg for either BMA or amniotic tissue groups displayed statistically significant improvement at 2 weeks ( 3.55 vs. $4.77, \mathrm{P}=0.002), 6$ months ( 2.34 vs. $3.37, \mathrm{P}=0.026)$, and 9 months (2.18 vs. $3.57, \mathrm{P}=0.01)$ (Fig. 5). Similarly, improvements in mean VAS-back were significant at 2 weeks ( 3.98 vs. 5.01, $\mathrm{P}=0.011), 2$ months ( 3.22 vs. $3.93, \mathrm{P}=0.04), 9$ months (2.38 vs. $4.11, \mathrm{P}=0.004)$, and 12 months ( 2.23 vs. $3.58, \mathrm{P}=$ 0.011) (Fig. 6).

There were statistically significant differences in the ODI ( 2 weeks, 42.19 vs. $31.11, \mathrm{P}=0.014)$ and SF-36 (4 months, 85

Table 1. Summary of Multiple Regression of OR Time on Selected Risk Factors

\begin{tabular}{|c|c|c|c|c|c|}
\hline & Estimate & Standard error & T value & $\operatorname{Pr}(>|\mathbf{t}|)$ & \\
\hline (Intercept) & 149.94 & 20.40 & 7.06 & $4.42 \times 10^{-11}$ & $* * *$ \\
\hline Age & 62.57 & 49.85 & 1.26 & 0.21 & \\
\hline BMI & 83.63 & 45.44 & 1.84 & 0.07 & $*$ \\
\hline $\mathrm{BMI}^{2}$ (squared) & 19.75 & 45.71 & 0.43 & 0.67 & \\
\hline DegenDiscYa & 38.28 & 13.76 & 2.78 & $<0.01$ & $* *$ \\
\hline 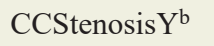 & 15.94 & 6.75 & 2.36 & 0.02 & $*$ \\
\hline
\end{tabular}

Significance codes: ***: 0; **: 0.001; *: 0.01; .: 0.05. Residual standard error: 43.63 on 166 degrees of freedom. Multiple R-squared: 0.3357; adjusted R-squared: 0.2917 . F-statistic: 7.628 on 11 and 166 DF, P value: $1.408 \times 10^{-10}$. OR: operating room. aDegenDiscY: presence of degenerative disc disease; ' $\mathrm{CCStenosisY:} \mathrm{presence} \mathrm{of} \mathrm{central} \mathrm{canal} \mathrm{stenosis.}$ 
Table 2. Summary of Multiple Regression of PACU Time on Selected Risk Factors

\begin{tabular}{|c|c|c|c|c|c|}
\hline & Estimate & Standard error & T value & $\operatorname{Pr}(>|t|)$ & \\
\hline (Intercept) & 4.81 & 0.07 & 71.46 & $<2 \times 10^{-16}$ & $* * *$ \\
\hline Age & 0.94 & 0.49 & 1.92 & 0.06 & * \\
\hline BMI & 0.28 & 0.49 & 0.58 & 0.56 & \\
\hline $\mathrm{BMI}^{2}$ (squared) & 0.03 & 0.49 & 0.05 & 0.96 & \\
\hline
\end{tabular}

Significance codes: **: 0; *: 0.01. Residual standard error: 0.4771 on 170 degrees of freedom. Multiple R-squared: 0.08937 ; adjusted R-squared: 0.05188 . F-statistic: 2.384 on 7 and 170 DF, P value: 0.02381 . PACU: postanesthesia care unit.

vs. $63, \mathrm{P}=0.043$ for amnion only), but these differences did not subsist over time (Figs. 6, 7).

\section{Complications}

There were no reportable intra-operative complications in this series of 269 patients. In two patients (one control, one amnion) re-herniation occurred, while there was a $0 \%$ re-herniation rate in the BMA and both patient groups.

\section{Discussion}

Neuroinflammation, which is associated with neuropathic pain, has key features. These include infiltration of immune cells into the peripheral nervous system such as the DRG, activation of microglia and astrocytes, and production of proinflammatory cytokines such as TNF, IL-1 $\beta$, and IL-6. Abundant research has suggested that this inflammatory response can be mediated by TGF- $\beta 1$. In addition, there is also evidence to suggest that the modulation of inflammation is possible by inhibiting monocyte infiltration, activation of the glia, and cytokine production in the DRG and spinal cord. Principally, it is through paracrine signaling that inflammation may be controlled by biological intervention.

Targeting the specific processes and molecules responsible for neuro-inflammation provides an opportunity for clinicians to exploit the inherent properties of orthobiologic products. Bone marrow derived stem cells have been examined in a number of applications for their ability to modulate neuroinflammation. They have been delivered via intra-ganglionic, intra-spinal, intra-brain, intra-muscular and intrathecal injection as well as through intravenous infiltration [19, 21-33].

One relevant study evaluated intrathecal delivered bone marrow-derived stem cells and their impact on neuropathic pain associated with constriction type injury. The study revealed that there was indeed an improvement in short and long term pain. There was also evidence that the dorsal root ganglion was protected from neuronal injury. It was proposed that the signaling mechanism was TGF- $\beta 1$ via a CXCR4/ CXCL12 interaction [20]. It is unclear whether TGF- $\beta 1$ from bone marrow-derived stem cells is the only source of TGF- $\beta 1$ that is effective at modulating the inflammation of the nerves.
Exogenous TGF- $\beta 1$ was administered in very low doses in the above study by Chen et al and found to be a potent analgesic [20]. This would suggest that other biologic therapies such as exosomes or ADPs that contain TGF- $\beta 1$ may also be effective. This is not to say that TGF- $\beta 1$ is the only cytokine that can modulate inflammation, but it is one in which an abundance of research has been done.

Furthermore, ADP has also proven an effective supplement, in addition to BMA, following micro-discectomies. ADP has been found to have the ability to decrease the amount of scar tissue formed post-operation through interrupting an important factor in the process of scar formation, TGF- $\beta 1$ signaling [34]. This theory was proven in rat models where it was found that not only did rats treated with human amniotic tissue have significantly less scar tissue, but they also showed a decrease in inflammatory cell infiltration and fibroblast proliferation [35]. In addition, it was found that dehydrated human amnion/chorion membrane decreased the amount of fibrosis in patients who underwent lumbar interbody lumbar fusion making epidural re-exploration easier [36]. These anti-inflammatory, anti-scarring, and anti-angiogenic properties of ADP come from a special heavy chain (HC)-hyaluronan (HA) complex purified from human amniotic membranes [37].

Disc re-herniation following micro-discectomy surgery remains a challenge that, until recently, remained unanswered. Rates of re-herniation vary between $2 \%$ and $10 \%$ according to a review of literature surrounding the topic of re-herniation after micro-discectomies between 2009 and 2015 [38]. In a recent study performed by Anderson et al [12], it was found that patients within the control group had re-herniation rates similar to prior studies, around $7.5 \%$. However, the patient group supplemented with ADP experienced a re-herniation rate of $0 \%$. These results are consistent with the findings of our current study and suggest that supplemented ADP is very beneficial in patient recovery and regeneration of vital structures surrounding the problem area following micro-discectomies. This result was improved upon when patients were supplemented with not only ADP, but also BMA, and resulted in a $0 \%$ re-herniation rate in our study.

Limitations to be acknowledged in the current study are several. First, this is a single-center study with all operations performed by a single investigator, although the surgical technique is fairly standardized for this type of endoscopic approach to microdiscectomy. Second, it is a single blinded 
prospective study with only the patient being blinded to the treatment. Thirdly, follow-up is only 12 months which is a medium-term outcome period, and longer observation period such as 5 years would be desirable. Finally, a larger multi-center clinical study should be performed.

\section{Conclusions}

Orthobiologic supplementation of BMA and ADP resulted in improved pain control during early post-operative periods. Results were measured by VAS, ODI and SF-36 scale surveys at the 2 weeks, 2 months, 4 months, 6 months, 9 months, and 12 months and showed significant improvement in comparison to standard (control) micro-discectomy post-surgery results. In this study, we had two patients who re-herniate, one in the control cohort and one in the ADP cohort in comparison to zero re-herniation seen in the ADP plus BMA cohort $(0 \%)$. These results show the strong potential for the application of $\mathrm{ADP}$ and BMA in the recovery and annular tissue regeneration in patients undergoing lumbar micro-discectomy. The patient population in this study demonstrates its safety, efficacy, and effectiveness in the treatment of annular tissue defects associated with lumbar microdiscectomy; however, larger studies are warranted to further validate our clinical findings.

\section{Acknowledgments}

The authors wish to thank the editorial board of Clinical Spine Surgery for review and criticism in improving the manuscript.

\section{Financial Disclosure}

The study was wholly self-funded by Spine Institute Northwest. There was no grant or outside funding source or sponsorship.

\section{Conflict of Interest}

None of the authors have conflict of interest to report.

\section{Informed Consent}

Informed consent was taken from patients prior to their participation in this randomized control trial.

\section{Author Contributions}

SK supervised the writing and submission of final draft of manuscript, has full access to all the data in the study, and takes responsibility for the integrity and the accuracy of the data analysis. DPS managed the literature searches and summaries of previous related work, and helped writing the first and final drafts of the manuscript.

\section{References}

1. Shriver MF, XieJJ TyeEY, et al. Limited microdiscectomy complication rates: A systematic review and meta-analysis. J Spinal Discord Tech. 2013;27:E8-E13.

2. Findlay GF, Hall BI, Musa BS, Oliveira MD, Fear SC. A 10-year follow-up of the outcome of lumbar microdiscectomy. Spine (Phila Pa 1976). 1998;23(10):1168-1171.

3. Dewing CB, Provencher MT, Riffenburgh RH, et al. The outcomes of lumbar microdiscectomy in a young, active population: correlation by herniation type and level. Spine. 2008;33:1.

4. Tseng SCG. HC-HA/PTX3 purified from amniotic membrane as novel regenerative matrix: insight into relationship between inflammation and regeneration. Invest Ophthalmol Vis Sci. 2015;56:1-8.

5. He H, Zhang S, Tighe S, Son J, Tseng SC. Immobilized heavy chain-hyaluronic acid polarizes lipopolysaccharide-activated macrophages toward M2 phenotype. J Biol Chem. 2013;288(36):25792-25803.

6. He H, Li W, Tseng DY, Zhang S, Chen SY, Day AJ, Tseng SC. Biochemical characterization and function of complexes formed by hyaluronan and the heavy chains of inter-alpha-inhibitor ( $\mathrm{HC} * \mathrm{HA})$ purified from extracts of human amniotic membrane. J Biol Chem. 2009;284(30):20136-20146.

7. Kamson S, Trescot AM, Sampson PD, Zhang Y. Fullendoscopic assisted lumbar decompressive surgery performed in an outpatient, ambulatory facility: report of 5 years of complications and risk factors. Pain Physician. 2017;20(2):E221-E231.

8. Ruetten S, Komp M, Merk H, Godolias G. Surgical treatment for lumbar lateral recess stenosis with the full-endoscopic interlaminar approach versus conventional microsurgical technique: a prospective, randomized, controlled study. J Neurosurg Spine. 2009;10(5):476-485.

9. Cong L, Zhu Y, Tu G. A meta-analysis of endoscopic discectomy versus open discectomy for symptomatic lumbar disk herniation. Eur Spine J. 2016;25(1):134-143.

10. Pan Z, Ha Y, Yi S, Cao K. Efficacy of Transforaminal Endoscopic Spine System (TESSYS) Technique in Treating Lumbar Disc Herniation. Med Sci Monit. 2016;22:530539.

11. Ruetten S, Komp M, Merk H, Godolias G. Full-endoscopic interlaminar and transforaminal lumbar discectomy versus conventional microsurgical technique: a prospective, randomized, controlled study. Spine (Phila $\mathrm{Pa}$ 1976). 2008;33(9):931-939.

12. Anderson G, Popov V, Raines A, et al. Cryopreserved amniotic membrane improves clinical outcomes following discectomy. ClinSpinal Surg. 2017;0:0.

13. Liu J, Sheha H, Fu Y, Liang L, Tseng SC. Update on amniotic membrane transplantation. Expert Rev Ophthalmol. 2010;5(5):645-661.

14. Bose B. Burn wound dressing with human amniotic membrane. Ann R Coll Surg Engl. 1979;61(6):444-447. 
15. Gruss JS, Jirsch DW. Human amniotic membrane: a versatile wound dressing. Can Med Assoc J. 1978;118(10):1237-1246.

16. Stern W. The grafting of preserved amniotic membranes to burned and ulcerated surfaces, substituting skin grafts. JAMA. 1913;60:973.

17. Li W, He H, Chen YT, Hayashida Y, Tseng SC. Reversal of myofibroblasts by amniotic membrane stromal extract. J Cell Physiol. 2008;215(3):657-664.

18. Tseng SC, Li DQ, Ma X. Suppression of transforming growth factor-beta isoforms, TGF-beta receptor type II, and myofibroblast differentiation in cultured human corneal and limbal fibroblasts by amniotic membrane matrix. J Cell Physiol. 1999;179(3):325-335.

19. Yousefifard M, Nasirinezhad F, Shardi Manaheji H, Janzadeh A, Hosseini M, Keshavarz M. Human bone marrow-derived and umbilical cord-derived mesenchymal stem cells for alleviating neuropathic pain in a spinal cord injury model. Stem Cell Res Ther. 2016;7:36.

20. Chen G, Park CK, Xie RG, Ji RR. Intrathecal bone marrow stromal cells inhibit neuropathic pain via TGF-beta secretion. J Clin Invest. 2015;125(8):3226-3240.

21. Musolino PL, Coronel MF, Hokfelt T, Villar MJ. Bone marrow stromal cells induce changes in pain behavior after sciatic nerve constriction. Neurosci Lett. 2007;418(1):97101.

22. Abrams MB, Dominguez C, Pernold K, Reger R, Wiesenfeld-Hallin Z, Olson L, Prockop D. Multipotent mesenchymal stromal cells attenuate chronic inflammation and injury-induced sensitivity to mechanical stimuli in experimental spinal cord injury. Restor Neurol Neurosci. 2009;27(4):307-321.

23. Siniscalco D, Giordano C, Galderisi U, Luongo L, Alessio N, Di Bernardo G, de Novellis V, et al. Intra-brain microinjection of human mesenchymal stem cells decreases allodynia in neuropathic mice. Cell Mol Life Sci. 2010;67(4):655-669.

24. Shibata T, Naruse K, Kamiya H, Kozakae M, Kondo M, Yasuda Y, Nakamura N, et al. Transplantation of bone marrow-derived mesenchymal stem cells improves diabetic polyneuropathy in rats. Diabetes. 2008;57(11):30993107.

25. Naruse K, Sato J, Funakubo M, Hata M, Nakamura N, Kobayashi Y, Kamiya H, et al. Transplantation of bone marrow-derived mononuclear cells improves mechanical hyperalgesia, cold allodynia and nerve function in diabetic neuropathy. PLoS One. 2011;6(11):e27458.

26. Zhang EJ, Song CH, Ko YK, Lee WH. Intrathecal administration of mesenchymal stem cells reduces the reactive oxygen species and pain behavior in neuropathic rats. Korean J Pain. 2014;27(3):239-245.

27. Li J, Deng G, Wang H, Yang M, Yang R, Li X, Zhang X, et al. Interleukin-1beta pre-treated bone marrow stromal cells alleviate neuropathic pain through CCL7-mediated inhibition of microglial activation in the spinal cord. Sci Rep. 2017;7:42260.

28. Fischer G, Wang F, Xiang H, Bai X, Yu H, Hogan QH. Inhibition of neuropathic hyperalgesia by intrathecal bone marrow stromal cells is associated with alteration of multiple soluble factors in cerebrospinal fluid. Exp Brain Res. 2017;235(9):2627-2638.

29. Klass M, Gavrikov V, Drury D, Stewart B, Hunter S, Denson DD, Hord A, et al. Intravenous mononuclear marrow cells reverse neuropathic pain from experimental mononeuropathy. Anesth Analg. 2007;104(4):944-948.

30. Siniscalco D, Giordano C, Galderisi U, Luongo L, de Novellis V, Rossi F, Maione S. Long-lasting effects of human mesenchymal stem cell systemic administration on painlike behaviors, cellular, and biomolecular modifications in neuropathic mice. Front Integr Neurosci. 2011;5:79.

31. Guo W, Chu YX, Imai S, Yang JL, Zou S, Mohammad Z, Wei F, et al. Further observations on the behavioral and neural effects of bone marrow stromal cells in rodent pain models. Mol Pain. 2016;12:1-12.

32. Guo W, Wang H, Zou S, Gu M, Watanabe M, Wei F, Dubner $\mathrm{R}$, et al. Bone marrow stromal cells produce longterm pain relief in rat models of persistent pain. Stem Cells. 2011;29(8):1294-1303.

33. Watanabe S, Uchida K, Nakajima H, Matsuo H, Sugita D, Yoshida A, Honjoh K, et al. Early transplantation of mesenchymal stem cells after spinal cord injury relieves pain hypersensitivity through suppression of pain-related signaling cascades and reduced inflammatory cell recruitment. Stem Cells. 2015;33(6):1902-1914.

34. Lee SB, Li DQ, Tan DT, Meller DC, Tseng SC. Suppression of TGF-beta signaling in both normal conjunctival fibroblasts and pterygial body fibroblasts by amniotic membrane. Curr Eye Res. 2000;20(4):325-334.

35. Choi HJ, Kim KB, Kwon YM. Effect of amniotic membrane to reduce postlaminectomy epidural adhesion on a rat model. J Korean Neurosurg Soc. 2011;49(6):323-328.

36. Subach BR, Copay AG. The use of a dehydrated amnion/ chorion membrane allograft in patients who subsequently undergo reexploration after posterior lumbar instrumentation. Adv Orthop. 2015;2015:501202.

37. Zhang S, Zhu YT, Chen SY, He H, Tseng SC. Constitutive expression of pentraxin 3 (PTX3) protein by human amniotic membrane cells leads to formation of the heavy chain (HC)-hyaluronan (HA)-PTX3 complex. J Biol Chem. 2014;289(19):13531-13542.

38. Anichini G, Landi A, Caporlingua F, Beer-Furlan A, Brogna C, Delfini R, Passacantilli E. Lumbar endoscopic microdiscectomy: where are we now? An updated literature review focused on clinical outcome, complications, and rate of recurrence. Biomed Res Int. 2015;2015:417801. 